\title{
Corrosive Behavior Study of Q235 Steel Coated with Epoxy Composites in Substation Environment
}

\author{
Yong $\mathrm{Li}^{1, \mathrm{a}}$, Jin $\mathrm{Fu}^{1, \mathrm{~b}}$, Wei Chen ${ }^{1, \mathrm{c}}$, Wenjing Yang ${ }^{2, \mathrm{~d}}$, Xueming $\mathrm{Li}^{2, \mathrm{e}}$, Yanjun Yin ${ }^{2, \mathrm{f}}$ \\ and Qihui Wang ${ }^{2, g}$ \\ ${ }^{1}$ State Grid Chongqing Electric Power Research Institute, Chongqing, China \\ ${ }^{2}$ College of Chemistry and Chemical Engineering, Chongqing University, Chongqing, China \\ aemail: hvliyong@163.com, bemail:fujin@cqu.edu.cn, ${ }^{c} e m a i l: c h e n w e i 1 @ c q u . e d u . c n,{ }^{d *}$ email: \\ yangwj308@163.com, eemail:xuemingli@cqu.edu.cn, 'email:yanjunyin@cqu.edu.cn, \\ gemail:qihuiwang@cqu.edu.cn
}

Keywords: Electrochemical impedance spectroscopy; Epoxy coating; Anti-corrosion.

Abstract. In this work, the epoxy composite coating composited by zinc rich epoxy primer and silver epoxy enamel, was sprayed on the surface of Q235 steel. The anticorrosion of as-prepared samples in substation environment with atmospheric exposure for 18 months, and in $5 \% \mathrm{NaCl}$ aqueous solution for 250 days were evaluated and compared, respectively. Electrochemical impedance spectroscopy (EIS) was employed to analyze the corrosive behavior of epoxy composite coating in $5 \% \mathrm{NaCl}$ aqueous solution, the weather resistance and salt resistance were also evaluated. Additionally, the structure changes of epoxy composite coating induced by salt immersion and atmospheric exposure were characterized by fourier transform infrared (FT-IR). The results are demonstrated that the epoxy composite coating is corroded to be invalid after immersed into $5 \% \mathrm{NaCl}$ aqueous solution for 250 days, which the impedance decreases from $3.967 \times 10^{9} \cdot \mathrm{cm}^{2}$ to $9.780 \times 10^{2} \cdot \mathrm{cm}^{2}$. The mechanism of corrosion is proposed that the corrosive medium gradually penetrate into the composite coating to form micro electric circuit through micro pores, resulting in the increase of porosity and corrosive rate for the composite coating. However, the impedance of epoxy composite coating in substation environment for 18 months is still $2.708 \times 10^{6} \cdot \mathrm{cm}^{2}$, indicating the good corrosion resistance.

\section{Introduction}

Recently, Q235 steel is applied to high voltage transmission tower of electric power system, bridges, vehicles, boilers, containers, ships widely [1]. The corrosive resistance of Q235 was improved by using anti-corrosion coating commonly [2]. The ethoxyline resin is considered to be a promising material due to their excellent mechanical properties and chemical resistance, easy crosslinking and curing to make film strong compactness, high adhesion [3-7]. The research of $\mathrm{H}$. Abd El-Wahab et al. [8] showed that a certain amount of polyamide can short the curing time of epoxy asphalt coating and the water resistance of coatings was also improved. Wang et al. [9] suggested that waterborne epoxy resin coatings were obtained dispersing nano- $\mathrm{SiO}_{2}$ into the single epoxy compound in the process of self curing agent, then in situ polymerization and grafting modification. In order to evaluate the corrosion resistance of epoxy coatings, Zhang et al. [10] studied the performance and failure process of organic coating-metal system and the electrochemical impedance spectroscopy of epoxy resin-steel system in $3.5 \% \mathrm{NaCl}$ aqueous solution was analyzed by electrochemical impedance spectroscopy (EIS), demanstrating the impedance was decreased in early immersion, increased in metaphase and then decreased in anaphase. The soaking time was prolonged, and the concentration of $\mathrm{Cl}$ - ion in the coating was increased.

In this work, the epoxy composite coating composited by zinc rich epoxy primer and silver epoxy enamel was sprayed on the surface of Q235 steel. Firstly, the as-prepared samples were immersion test in $5 \% \mathrm{NaCl}$ aqueous solution or corroded through atmospheric exposure test 
in substation environment for different time respectively. Then, EIS was employed to analyze the corrosive behavior of epoxy composite coating to evaluate the weather resistance and salt resistance. Additionally, the structure changes of epoxy composite coating treated by salt immersion and atmospheric exposure were characterized by fourier transform infrared (FT-IR).

\section{Experiment}

H06-4 zinc rich epoxy primer and silver epoxy enamel was supplied by Chongqing Three Gorges paint Limited by Share Ltd.. In order to get the roughness of Sa 2.5, Q235 (250 mm×150 mm×1.8 mm) was pretreated by cast steel shot blasting primarily. However, it is necessary to finish the coating treatment of primer within $2 \mathrm{~h}$ to avoid the substrate oxidation. Significantly, primer and topcoat were coated on Q235 orderly by spraying and etch coating should be coated for two times with the two layers vertically to etch other. The primer coating was dried for $4-8 \mathrm{~h}$ before topcoat coating. The film thickness of primer and topcoat is approximately to $80 \mu \mathrm{m}$ and $70 \mu \mathrm{m}$ respectively. Moreover, the back of coating sample with excellent weather resistance was used to edge banding with the width of $5 \mathrm{~mm}$ after the final coating is completely dried. Finally, sample preparation was completed after being dried naturally and status adjustment disposal placed in the conditions specified in GB 9278 for seven days.

In the experiment, the as-prepared samples were treated by immersion using $5 \% \mathrm{NaCl}$ aqueous solution and natural exposure handled in $110 \mathrm{kV}$ substation environment without industrial and enterprise, respectively. For the evaluation of related performance, the treated samples were taken out termly to be analyzed by EIS.

After immersed in $5 \% \mathrm{NaCl}$ aqueous solution for $2 \mathrm{~h}$, the coating sample was tested in shielding box with the open circuit potential of sine wave signal amplitude $10 \mathrm{mV}$ and frequency range $10^{-2}-10^{5}$ Hz. The PGSTAT 302 electrochemical testing system with three electrode system was employed to testing EIS. Coating sample was used as working electrode (analyzed area $12.56 \mathrm{~cm}^{2}$ ), graphite and $\mathrm{Ag} / \mathrm{AgCl}$ were regarded as counter electrode and reference electrode, respectively.

\section{Results and Discussion}

\section{The weather resistance of epoxy composite coating}

The surface picture of epoxy composite coating exposured in nature for 18 months was shown in Fig.1. No pitting, cracks and corrosion marks can be found and the surface is smooth in the coating sample, indicating the good weather resistance of epoxy composite coating. In order to understand the corrosive properties of coating sample deeply, the Fig.2 showed the electrochemical impedance spectroscopy of epoxy composite coating exposured for different time. The equivalent circuit diagram $3 \mathrm{a}$ is used to fit with Zview software to obtain Rct and Cdl in Table 1.

The impedance of coatings decreases gradually with the exprosure time prolonged, indicating the corrosion resistance of the coating was gradually decreased. The impedance of coatings is $2.708 \times 10^{6}$ $\Omega \cdot \mathrm{cm}^{2}$, demonstrating the good protection of coating, which is consistent with the results of Fig.5.

Moreover, the FT-IR result illustrates that each characteristic peak is weakened obviously without any peak shift and new peak after atmospheric exposure experiment of epoxy coatings for different time, which is shown in Fig.3. Speculatively, the performance decay of epoxy coating happened in the exposure experiment. It is because of a series of broken bonds and redox reactions occurring due to the production of small molecular substances or being washed away by rain on the surface of epoxy composite coating in the effect of sunlight, temperature, moisture. 

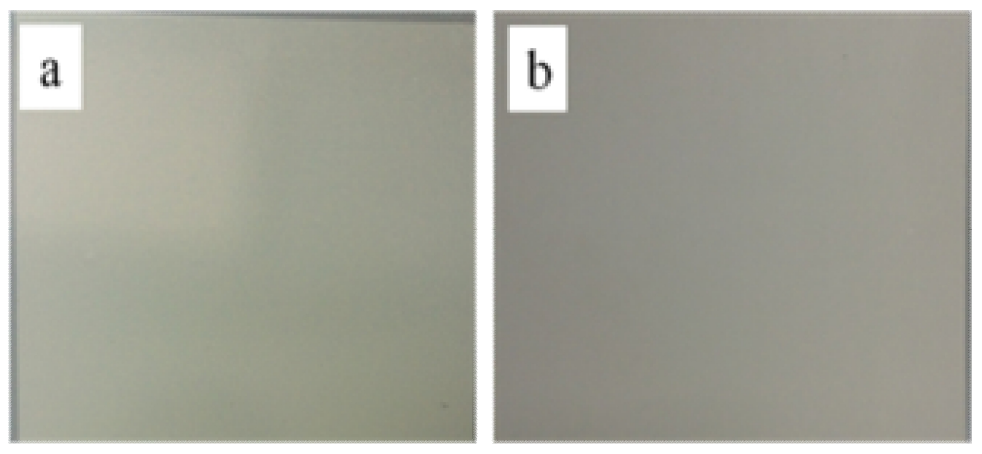

Fig.1 Optics pictures of Q235 epoxy composite coating (a) without atmospheric exposure and (b) atmospheric exposure test for 18 months.
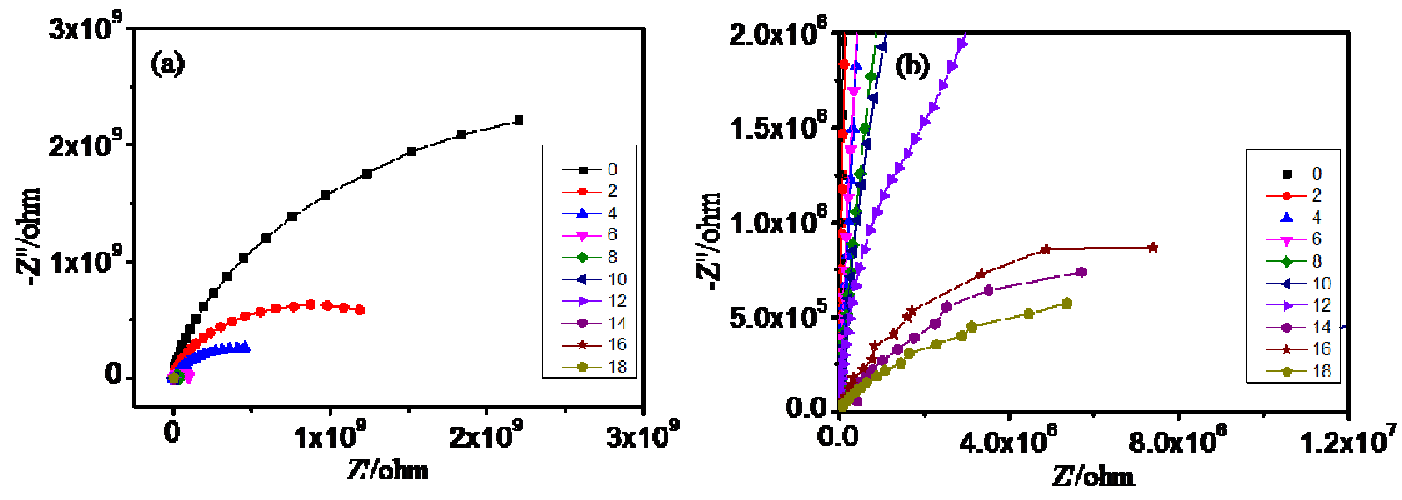

Fig.2 EIS results of Q235 epoxy composite coating in different atmospheric exposure time

Table.1 Resistance and capacitance of the Q235 epoxy composite coating in different atmospheric exposure time

\begin{tabular}{ccc}
\hline Time/Month & $\mathrm{Rct} / \Omega \cdot \mathrm{cm}^{2}$ & $\mathrm{C}_{\mathrm{dl}} / \mathrm{F} \cdot \mathrm{cm}^{-2}$ \\
\hline 0 & $3.967 \times 10^{9}$ & $2.207 \times 10^{-9}$ \\
2 & $1.052 \times 10^{9}$ & $2.702 \times 10^{-9}$ \\
4 & $5.346 \times 10^{9}$ & $6.966 \times 10^{-9}$ \\
6 & $1.205 \times 10^{8}$ & $9.206 \times 10^{-9}$ \\
8 & $5.100 \times 10^{7}$ & $2.162 \times 10^{-8}$ \\
10 & $1.313 \times 10^{7}$ & $3.217 \times 10^{-8}$ \\
12 & $6.502 \times 10^{6}$ & $3.965 \times 10^{-8}$ \\
14 & $4.899 \times 10^{6}$ & $6.155 \times 10^{-8}$ \\
16 & $3.936 \times 10^{6}$ & $5.202 \times 10^{-8}$ \\
18 & $2.708 \times 10^{6}$ & $1.903 \times 10^{-8}$ \\
\hline
\end{tabular}




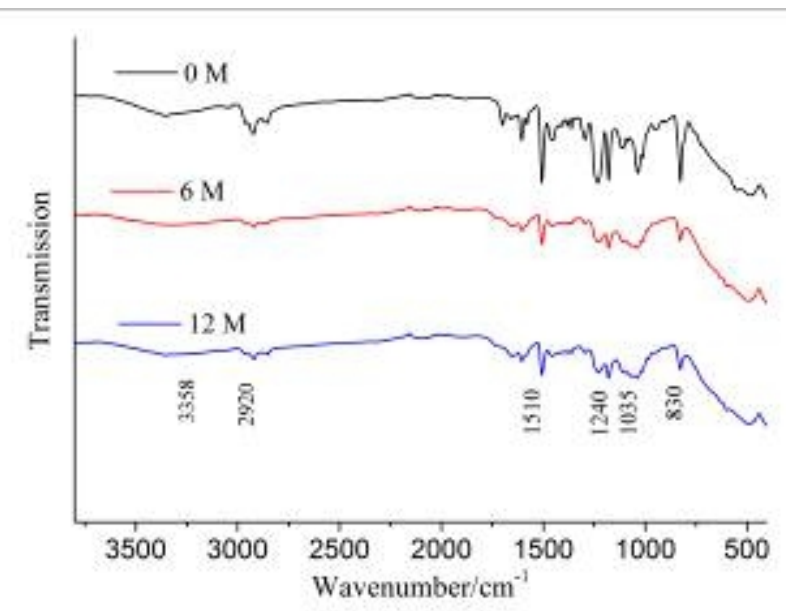

Fig.3 FT-IR spectra of the Q235 epoxy composite coating in different atmospheric exposure time

\section{The salt resistance of epoxy composite coating}

In order to evaluate the salt resistance, the Q235 steel coated with epoxy composite was immersed into $5 \% \mathrm{NaCl}$ aqueous solution for 0,90 and 250 days and the corresponding digital photographs are shown in Fig.4. It is found that the bubbles of the coating increase a lot and become bigger with time going, and the coating damage is worse with the increase of immersing time. The surface of epoxy coating is smooth without immersing in Fig.4a, and the bubbles appear when the immersion time reach to 90 days in Fig.4b. Moreover, a large amount of bubbles with cracks and rust are observed after being immersed for 250 days in Fig.4c. The reason is that solution penetrate into the composite coating through micro pores gradually, resulting in the electrochemical corrosion reaction and constant corrosion product reaching into the surface of coating through micro-porous.
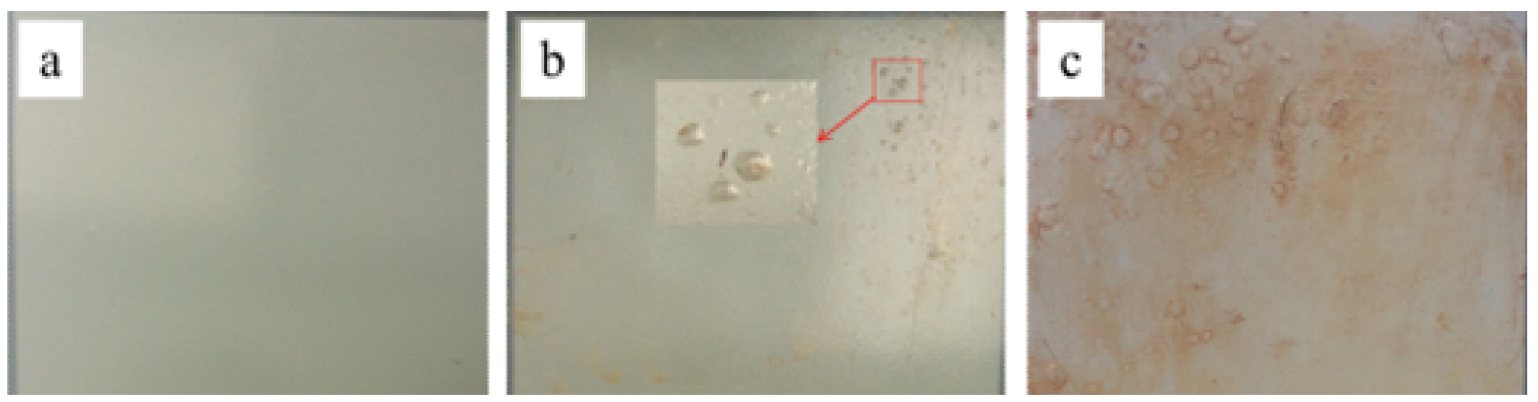

Fig.4 Optics pictures of Q235 epoxy composite coating with different immersion time (a) 0 day, (b)

90 days and (c) 250 days
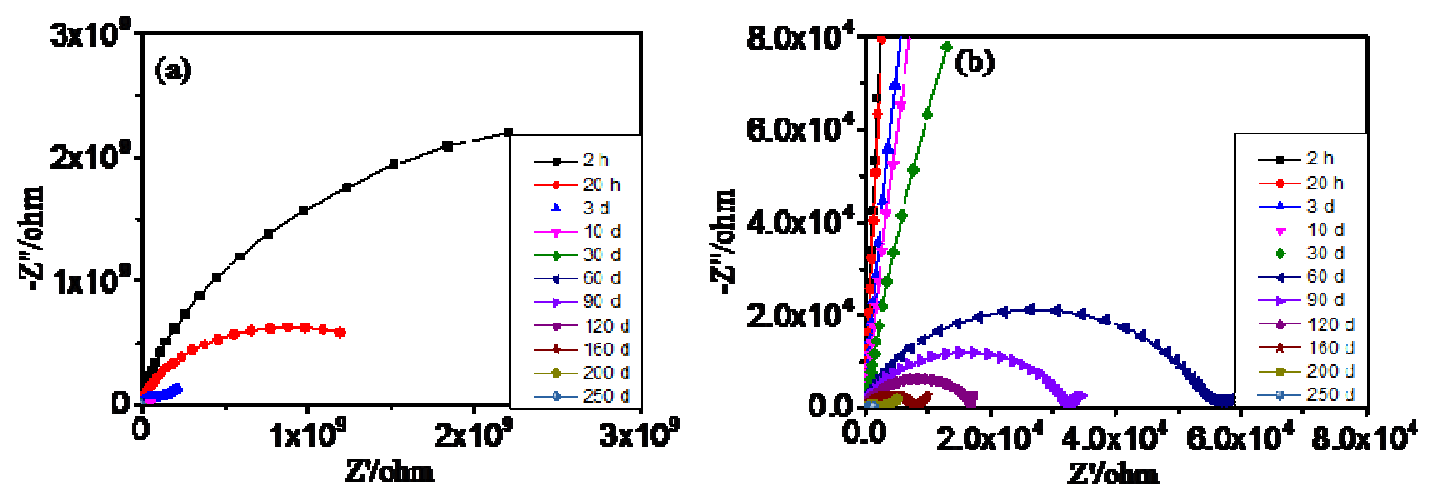

Fig.5 EIS results of Q235 epoxy composite coating with different immersion time 
(a)

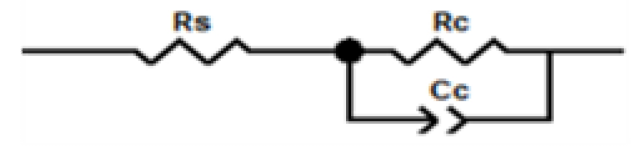

(b)

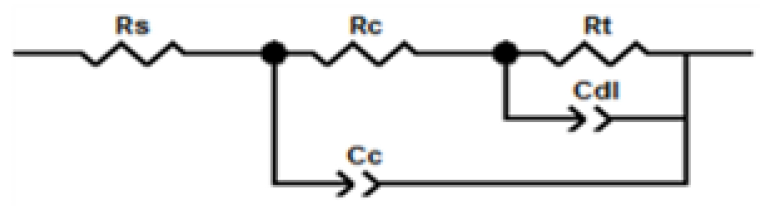

Fig.6 Equivalent circuit of EIS

The EIS results of epoxy composite coating treated in $5 \% \mathrm{NaCl}$ aqueous solution for different time were revealed in Fig.5. Fig.5b is the local magnification of Fig. 5a. In the primeval immersion, the EIS only shows a time characteristic constant by fitting Z-view software using equivalent circuit diagram 3a. At this time, the resistance of coating is much large, which is isolated from the substrate and corrosive media contact, playing a protective role. A small arc resistance in high frequency region and a large arc resistance in low frequency region appear in Nyquist spectrum and there are two time characteristic constants in EIS after immersing 3 days, illustrating that electrolyte penetrated into the coating with the effects of penetration to reach the surface of the metal substrate. After that, the coating enters into medium and late stages of immersion. The equivalent circuit diagram $6 \mathrm{~b}$ is used to fit with Zview software to obtain Rct and Cdl in Table 2.

\begin{tabular}{ccc}
\hline Time/Month & Rct $/ \Omega \cdot \mathrm{cm}^{2}$ & $\mathrm{C}_{\mathrm{dl}} / \mathrm{F} \cdot \mathrm{cm}^{-2}$ \\
\hline $2 \mathrm{~h}$ & $3.967 \times 10^{9}$ & $2.207 \times 10^{-9}$ \\
$20 \mathrm{~h}$ & $1.276 \times 10^{9}$ & $2.522 \times 10^{-9}$ \\
$3 \mathrm{~d}$ & $1.845 \times 10^{8}$ & $3.618 \times 10^{-9}$ \\
$10 \mathrm{~d}$ & $3.385 \times 10^{7}$ & $4.515 \times 10^{-9}$ \\
$30 \mathrm{~d}$ & $1.715 \times 10^{6}$ & $5.954 \times 10^{-9}$ \\
$60 \mathrm{~d}$ & $5.367 \times 10^{4}$ & $1.653 \times 10^{-8}$ \\
$90 \mathrm{~d}$ & $3.215 \times 10^{4}$ & $2.413 \times 10^{-8}$ \\
$120 \mathrm{~d}$ & $1.700 \times 10^{4}$ & $3.496 \times 10^{-8}$ \\
$160 \mathrm{~d}$ & $8.233 \times 10^{3}$ & $9.337 \times 10^{-8}$ \\
$200 \mathrm{~d}$ & $1.908 \times 10^{3}$ & $5.676 \times 10^{-8}$ \\
$250 \mathrm{~d}$ & $9.780 \times 10^{2}$ & $2.522 \times 10^{-7}$ \\
\hline
\end{tabular}

Table.2 Resistance and capacitance of the Q235 epoxy composite coating with different immersion time

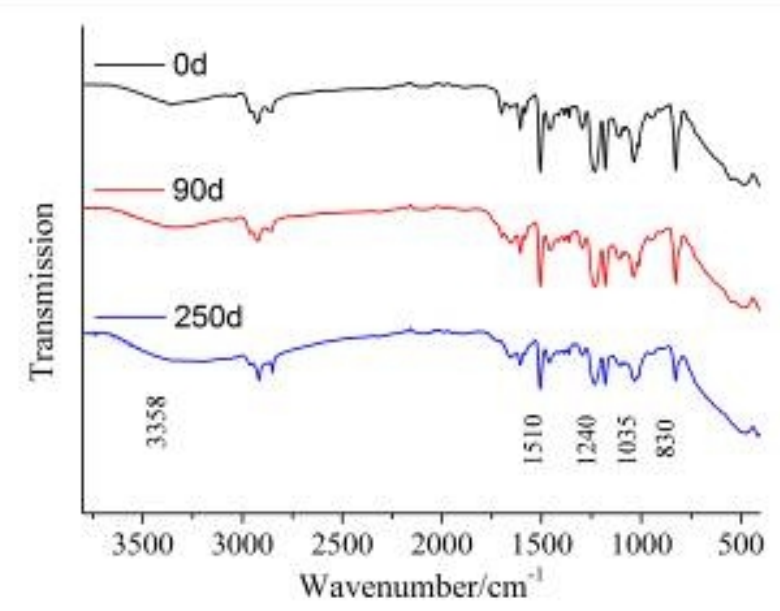

Fig.7 FT-IR spectra of Q235 epoxy composite coating in different immersion time. 
Analyzed from Table 2, the coating resistance decreases with the increase of immersion time and the $\mathrm{Cdl}$ increases gradually since the porosity of coating increase with the increasing of immersion time and corrosive medium is gradually permeated into the composite coating to form micro electric circuit through micro pores.

Research shows that metal corrosion under the coating has been carried out when the resistance of the coating Rct value less than $10^{6} \Omega \cdot \mathrm{cm}^{2}$ and the coating is considered to be disabled when the Rct value is less than $10^{3} \Omega \cdot \mathrm{cm}^{2}$ [11]. From table 2, the resistance of coating is $1.715 \times 10^{6} \Omega \cdot \mathrm{cm}^{2}$ and $5.367 \times 10^{4} \Omega \cdot \mathrm{cm}^{2}$ immersed for 30 days and 60 days respectively. It is considered that the corrosive medium reaches the surface of the substrate, and the substrate is corroded after 30 days immersion. In addition, the coating is determined to be failure when the resistance decreases to $9.780 \times 10^{2} \Omega \cdot \mathrm{cm}^{2}$ immersing for 250 days.

After immersed for 0 days, 90 days and 250 days in $5 \% \mathrm{NaCl}$ aqueous solution, the FI-IR results of epoxy composite coating were shown in Fig.7. Obviously, $3358 \mathrm{~cm}^{-1}$ is the $-\mathrm{OH}$ characteristic absorption peak and the peak of $-\mathrm{NH} 2,1510 \mathrm{~cm}^{-1}$ is $\mathrm{C}-\mathrm{N}$ vibration absorption peak and the characteristic absorption peak of ether bond corresponds to $1240 \mathrm{~cm}^{-1}$ and $1035 \mathrm{~cm}^{-1}$. It is probable the ether bond and $\mathrm{C}-\mathrm{N}$ bond were hydrolyzed to $-\mathrm{OH}$ and $-\mathrm{NH} 2$ in the process of immersion to cause the coating to be failure.

\section{Conclusion}

In this study, the epoxy composite coating was treated by atmospheric natural exposure and exposure in $5 \% \mathrm{NaCl}$ aqueous solution to evaluate the weather resistance and salt resistance using EIS method. There is degradation reaction of organic matter during exposure in nature causing the protective effect decreased of coatings. However, the resistance of coating is $2.708 \times 10^{6} \Omega \cdot \mathrm{cm}^{2}$ after exposure for 18 months and the coating is not disable with excellent weather resistance. When coating was immersed into $5 \% \mathrm{NaCl}$ aqueous solution, the ether bond and $\mathrm{C}-\mathrm{N}$ bond were hydrolyzed, decreasing the protective properties of the coating on the metal substrate. The expoy coating was disable after immersing for 250 days with the decrease of resistance from $3.967 \times 10^{9} \Omega \cdot \mathrm{cm}^{2}$ to $9.780 \times 10^{2} \Omega \cdot \mathrm{cm}^{2}$.

\section{References}

[1]X. Li, Y.T. Li, H. S. Jiang. Journal of Materials Protection, 45 (2012):28-31.

[2] H.J. Cao, X. Zhang, J. Han.Materials China, 33 (2014): 20-25.

[3] S. J. Park, M. H. Kim, J. R. Lee. J. Colloid Interf. Sci. 228 (2000): 287-291.

[4] S. M. Mousavifard, P. M. Nouri, M. Attar. J. Ind. Eng. Chem. 19 (2013): 1031-1039.

[5] Y. Li, P. Badrinarayanan, M. R. Kessler. Polymer, 2013, 54 (12):3017-3025.

[6] L. L. Lin, T. H. Ho, C. S. Wang. Polymer. 38 (1997): 1997-2003.

[7] S. W. Suh, J. J. Kim, S. H. Kim. J. Ind. Eng. Chem. 18 (2012):290-294.

[8] H. A. El-Wahab, A. M. Saleh, M. A. Wassel. Prog. Org. Coat. 76 (2013): 1363-1368.

[9]Y.T. Wang, T.B. Wang, S.Y. Gu. Journal of Building Materials, 10 (2006): 677-681.

[10]Y. H. Zhang, L.N. Xu, M. X. Lu. Corrosion and Protection, 28 (2007):227-234.

[11] J. D. Scantlebury, K. Gali,. Prog. Org. Coat. 31 (1997): 201-207. 